\title{
Selective Laser Sintering of PA6: Effect of Powder Recoating on Fibre Orientation
}

\author{
Tobias Heckner ${ }^{1, *}$, Michael Seitz ${ }^{1}$, Sven Robert Raisch ${ }^{1}{ }^{\mathbb{D}}$, Gerrit Huelder ${ }^{1}$ and \\ Peter Middendorf ${ }^{2}$ \\ 1 Robert Bosch GmbH, Corporate Research, 71272 Renningen, Germany; \\ fixed-term.michael.seitz4@bosch.com (M.S.); svenrobert.raisch@de.bosch.com (S.R.R.); \\ gerrit.huelder@de.bosch.com (G.H.) \\ 2 Institute of Aircraft Design, University of Stuttgart, 70569 Stuttgart, Germany; \\ middendorf@ifb.uni-stuttgart.de \\ * Correspondence: tobias.heckner@de.bosch.com
}

Received: 30 June 2020; Accepted: 1 August 2020; Published: 6 August 2020

\begin{abstract}
In Selective Laser Sintering, fibres are strongly orientated during the powder recoating process. This effect leads to an additional increase of anisotropy in the final printed parts. This study investigates the influence of process parameter variation on the mechanical properties and the fibre orientation. A full factorial design of experiment was created to evaluate the processing parameters of recoating speed, layer thickness and laser power on the part's modulus of elasticity. Based on the mechanical testing, computed tomography was applied to selected samples to investigate the process-induced fibre microstructure, and calculate the fibre orientation tensors. The results show increasing part stiffness in the deposition direction, with decreasing layer thickness and increasing laser power, while the recoating speed only shows little effect on the mechanical performance. This complies with computed tomography imaging results, which show an increase in fibre orientation with smaller layer thickness. With thinner layers, and hence smaller shear gaps, shear stresses induced by the roller during recoating increase significantly, leading to excessive fibre reorientation and alignment. The high level of fibre alignment implies an increase of strength and stiffness in the recoating direction. In addition, thinner layer thickness under constant laser energy density results in improved melting behaviour, and thus improved fibre consolidation, consequently further increasing the mechanical properties. Meanwhile, the parameters of recoating speed and laser power do not have a significant impact on fibre orientation within their applicable process windows.
\end{abstract}

Keywords: selective laser sintering; recoating; PA6; polyamide; glass; fibres; beads; orientation; recoating speed; layer thickness; energy density

\section{Introduction}

In polymer and composite technologies, reinforcement fillers of various materials are commonly used to increase part strength and stiffness. In injection molding, it is well known that fibres align inside the melt flow and display characteristic fibre orientation patterns inside the center of the flow and outer shell [1]. In addition to fibre orientation, fibre content distribution and length can vary within different locations inside a part [2]. Due to the effect of fibre matrix separation, areas within a part can appear with significant deviations in filler content [3].

In Selective Laser Sintering (SLS), part strength and stiffness can also be increased with the use of fibre-reinforced powders. While the typical layer-by-layer build-up differs greatly from the injection molding process, changes in filler orientation and part anisotropy are also observed. This is mainly caused by fibre alignment during recoating. 
Jansson and Pejryd [4] investigated the orientation of carbon short fibres in SLS processing. Their results show greater fibre alignment in the direction of powder recoating, while longer fibres exhibit higher levels of orientation. During recoating, the recoating blade touches longer fibres and aligns them into recoating direction, while short fibres with a length below the layer thickness align more randomly. This investigation is supported by Arai et al. [5], who determined the anisotropy of glass fibres in polybutylene terephthalate (PBT). Arai showed that the tensile strength and modulus of elasticity show their highest values in the recoating direction, and lowest mechanical properties perpendicular to it. Furthermore, Jansson and Pejryd [2] and Schmid [6] state that fibre-reinforced SLS parts can show worse mechanical properties in the perpendicular build-up direction than unreinforced parts with equal polymer materials. This is caused by reduced fibre orientation in the thickness direction, and hence lower layer-to-layer connection [4].

In addition, the influence of powder recoating and the energy density on the mechanical properties of unreinforced SLS material was investigated. Beitz [7] compared different recoating systems, such as roller and blade. He found that the recoating unit applies pressure onto the powder bed, dependent on the recoating speed and type of recoating unit. Drexler et al. [8] showed no significant effects on part density with different recoating speeds, but an impact on the mechanical properties can be observed. Tensile strength as well as elongation at break show the highest values for the lowest recoating speed. This effect is explained via the longer interaction time of the recoating unit with the powder particles. As a consequence, a more homogenous part surface results.

The influence of laser energy density was previously studied for unreinforced materials [9]. Lanzl et al. [10] conducted the first experiments to investigate the effect of the energy density on the mechanical properties of fibre-reinforced materials. In their investigations, fibre content was varied from 10 to 50 vol.\%, while particularly fibre contents of up to 30 vol.\% showed a high impact on the modulus of elasticity. The variation of energy density from 0.04 to $0.06 \mathrm{~J} / \mathrm{mm}^{2}$ had only a little influence on part performance. At very low energy density levels, the modulus of elasticity decreased significantly due to the low melting behaviour of the polymer particles. These results are supported by Arai's et al. [5] investigation. The authors investigated the orientation of glass fibres in a PBT SLS material. At a layer thickness of $100 \mu \mathrm{m}$, the tensor shows an orientation of $50 \%$ in the recoating direction and $35 \%$ in the cross direction, while the build-up direction $\mathrm{Z}$ displays only $15 \%$. This behaviour is also observed in the mechanical properties, whereas the highest tensile strength and modulus of elasticity were found in the recoating direction, while the lowest properties were found in the build-up direction. Furthermore, it was found that the samples in the coating direction showed the highest heat deflection temperature and the lowest shrinkage effects.

Besides the process influence, the powder and fibre composition also have an influence on fibre orientation and part behaviour. Zhu et al. [11] developed a method whereby a green body manufactured with SLS is combined with epoxy resin infiltration. After the modification of carbon fibres (CF) with a Polyamide 12 (PA12) surface treatment, the PA12/CF composite powder was used for the SLS process. This method enables a better random three-dimensional distribution of the fibres.

The literature review shows that prior studies investigated the mechanical properties as a consequence of fibre orientation. The characterisation of fibre orientation is only described by different sample orientations in the build job. Therefore, this study describes the fibres' orientation by calculation of the orientation tensors in the $\mathrm{X}, \mathrm{Y}$ and $\mathrm{Z}$ direction. In this context, the impact of the processing conditions on the fibre orientation tensor is described. The parameters of recoating speed, layer thickness and laser power are varied, and the interactions between these three parameters are identified in this study.

\section{Materials and Methods}

This research explores the influence of process parameters on fibre orientation. Based on a literature review, an experimental design was created to identify the influence of processing parameters on the fibre orientation. After identifying a suitable sintering window, samples were manufactured according 
to the experimental design and chosen parameters. The results of mechanical testing are used to identify samples with remarkable mechanical performance, to be investigated by means of X-ray computed tomography (CT).

\subsection{Design of Experiment}

A full factorial design of experiment (DoE) with the parameters of recoating speed (RS), layer thickness (LT) and laser power (LP) was created. The parameters were identified based on a literature review, as mentioned earlier. For each parameter, three factor levels were set: low, medium and high. The bottom and top limit values were maximised in order to observe high effects on the fibre orientation. Although going to the process limits, safe processing had to be ensured, characterised by low curling and warpage effects. The values of the DoE are summarised in Table 1. All other processing parameters were kept constant. Three parameters with three levels leads to a $3^{3}$ DoE.

Table 1. Varied parameters for effect identification on fibre orientation.

\begin{tabular}{cccc}
\hline Level & $\begin{array}{c}\text { Recoating Speed } \\
\text { in } \mathbf{~} \mathbf{m} / \mathbf{s}\end{array}$ & $\begin{array}{c}\text { Layer Thickness } \\
\text { in } \boldsymbol{\mu \mathbf { m }}\end{array}$ & $\begin{array}{c}\text { Laser Power } \\
\text { in } \mathbf{W}\end{array}$ \\
\hline Low & 168 & 100 & 16 \\
Medium & 228 & 120 & 26 \\
High & 288 & 140 & 36 \\
\hline
\end{tabular}

\subsection{Manufacturing of Samples}

For the manufacturing of samples, a HT252P SLS printer made by Farsoon (Hunan, China), was applied. The printer features a maximum chamber temperature of $220^{\circ} \mathrm{C}$ and is equipped with a $60 \mathrm{~W} \mathrm{CO}_{2}$ laser (wavelength $10.6 \mu \mathrm{m}$ ). The build chamber is heated from all side: from the top by infrared radiation (IR) heaters, and from the cylinder walls and piston plate by integrated heating bands. The surface of the powder reservoir is heated by IR heaters. In addition, the chamber is flooded with nitrogen during the complete build job, leading to a residual oxygen content below $0.3 \%$ throughout all build stages. Prior to the heat-up stage, the machine was flooded manually with nitrogen and heated up to $150{ }^{\circ} \mathrm{C}$ for $1 \mathrm{~h}$. After this period, the actual warm-up stage began. The warm-up stage started with a ramp from the start temperature of $150{ }^{\circ} \mathrm{C}$ up to the final build temperature. The optimum build temperature was identified prior to testing by differential scanning calorimetry (DSC) analysis, supplier recommendations and printing pre-trials. The cylinder, piston and feed temperatures had been optimised in pre-tests with the target of minimizing curling and warpage effects. After the core temperature of the part cake had cooled down to below $50{ }^{\circ} \mathrm{C}$, samples were removed and cleaned. As the impact by blasting could lead to uneven surfaces, the removal of the remaining powder from the specimens was done manually with a brush, and additionally with a vacuum cleaner with an attached brush.

Based on the DSC analysis (Section 3.1) and knowledge from pre-trials, the parameters for the temperatures were identified as summarised in Table 2. The temperature of the cavity walls (cylinder temperature) and piston plate was set to $180^{\circ} \mathrm{C}$. The surface temperature (build temperature) of the powder after recoating was set to $210^{\circ} \mathrm{C}$. While RS, LT and LP were changed according to the DoE, the temperatures, the scanning speed and the hatch distance were kept constant.

For tensile testing, tensile bars according to DIN EN ISO 527-2 1A were printed. For CT scans, cubes with a geometry of $3 \mathrm{~mm} \times 3 \mathrm{~mm} \times 3 \mathrm{~mm}$ on a base platform were chosen. The base allowed labelling as well as easier positioning on the CT platform. Both samples are illustrated in Figure 1.

\subsection{Investigated PA6 Material}

A non-commercial Polyamide 6 (PA6) powder, supplied by Farsoon (FS6140GF), filled with 30 wt.\% glass beads and 10 wt.\% glass fibres, was used for this study. Due to the effects whereby fibres increase the anisotropy and decrease the flowability of the powder, beads facilitate the powder 
recoating, the isotropy and the warpage behaviour. The reinforcing fillers were added to the powder and were not integrated into the powder particles. The particle size distribution was D50 $=63 \mu \mathrm{m}$. Carbon black was added as colourant as well as additives for oxidation resistance. The melting point was $225^{\circ} \mathrm{C}$ and the bulk density of the powder was $0.6-0.73 \mathrm{~g} / \mathrm{cm}^{3}$, while the density of the printed parts was $1.4 \mathrm{~g} / \mathrm{cm}^{3}$ at sufficient energy input.

Table 2. Processing parameters of PA6-GB-GF (Farsoon FS6140GF).

\begin{tabular}{ccccc}
\hline Parameter & Unit & \multicolumn{3}{c}{ Value } \\
\hline Recoating Speed & $\mathrm{mm} / \mathrm{s}$ & 168 & 228 & 288 \\
\hline Layer Thickness & $\mu \mathrm{m}$ & 100 & 120 & 140 \\
\hline Build Temperature & ${ }^{\circ} \mathrm{C}$ & & 210 & \\
\hline Cylinder Temperature & ${ }^{\circ} \mathrm{C}$ & 180 & \\
\hline Piston Temperature & ${ }^{\circ} \mathrm{C}$ & 180 & \\
\hline Feed Temperature & ${ }^{\circ} \mathrm{C}$ & 165 & \\
\hline Scanning Speed Fill & $\mathrm{m} / \mathrm{s}$ & & 10.16 & \\
\hline Hatch distance & $\mathrm{mm}$ & & 0.15 & 36 \\
\hline Power Fill & $\mathrm{W}$ & 16 & 26 &
\end{tabular}

Tensile Bar

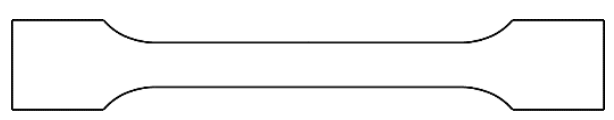

According to DIN EN ISO 527-2 1A.

(a)

\section{CT Cube}

Powder Recoating

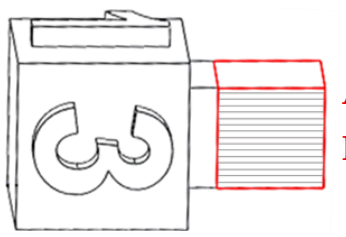

Area of

Measurement

(b)

Figure 1. Overview of the manufactured samples. (a) Tensile bars to identify the mechanical properties and (b) computed tomography $(\mathrm{CT})$ cubes to investigate the fibre orientation.

\subsection{Mechanical Testing and Fibre Orientation Determination}

Before the determination of the mechanical properties, the samples were vacuum dried at $80{ }^{\circ} \mathrm{C}$ for $168 \mathrm{~h}$. The modulus of elasticity was measured with an extensometer at $1 \mathrm{~mm} / \mathrm{min}$ according to DIN EN ISO 527-2/1A/5. After determining the linear-elastic strain area, the testing speed was increased to $5 \mathrm{~mm} / \mathrm{min}$. For the determination of the tensile properties, a Z050 by Zwick Roell (Ulm, Germany), with an extensometer was used. The measurements were performed under environmental conditions at room temperature of $23^{\circ} \mathrm{C}$ and humidity of $50 \%$.

$\mathrm{X}$-ray computer tomography was applied for investigation of the fibre orientation and to collect images for qualitative and quantitative analysis. A v|tome|x m CT machine by GE Inspection Technologies, United States, with a resolution of $2 \mu \mathrm{m}$ for detailed measuring range and $6 \mu \mathrm{m}$ for overview images, was used. Figure 2 illustrates the high resolution area (green). The lower resolution is only needed for identification of the labels and positioning of the samples for calculation analysis.

The software VG Studio Max allows the detection of fibre orientation and evaluation of the differences due to different processing parameters. For the calculation of fibre orientation, a cube of $2.8 \mathrm{~mm} \times 2.8 \mathrm{~mm} \times 2.8 \mathrm{~mm}$ inside the CT cube $(3 \mathrm{~mm} \times 3 \mathrm{~mm} \times 3 \mathrm{~mm})$ was defined and analysed, as shown in Figure 3a. This way, typical SLS effects at the edge zones, such as shrink marks, are neglected, which increases the comparability of the samples. The cube (blue coloured) was further sliced into 
21 layers $(1 \times 1 \times 21, X \times Y \times Z$ mesh $)$ as illustrated in Figure $3 b$. Thus, a better understanding of the fibre orientation through the single layers could be obtained.

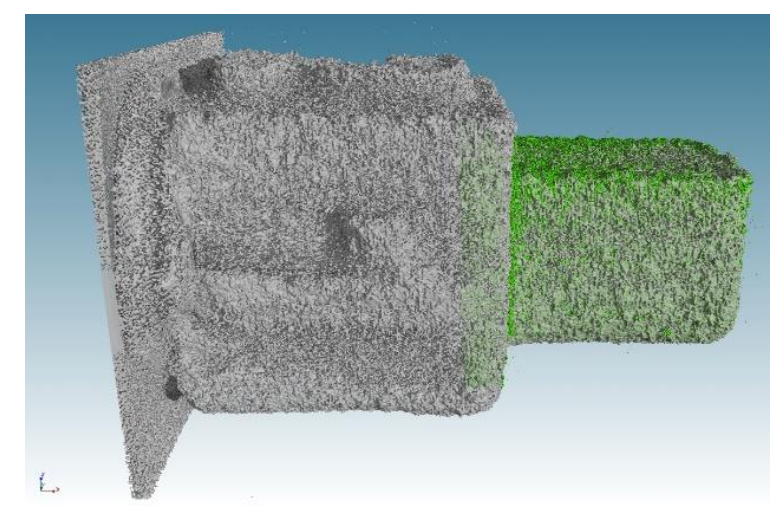

Figure 2. Illustration of the CT resolution within the CT cube. Green represents the high detail area with a resolution of $2 \mu \mathrm{m}$.

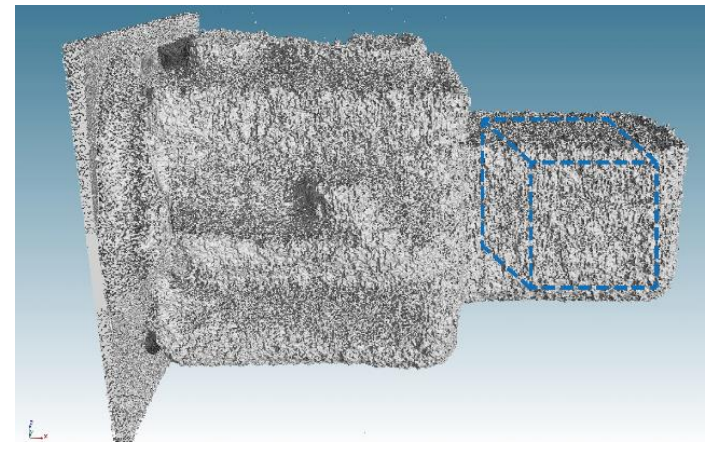

(a)

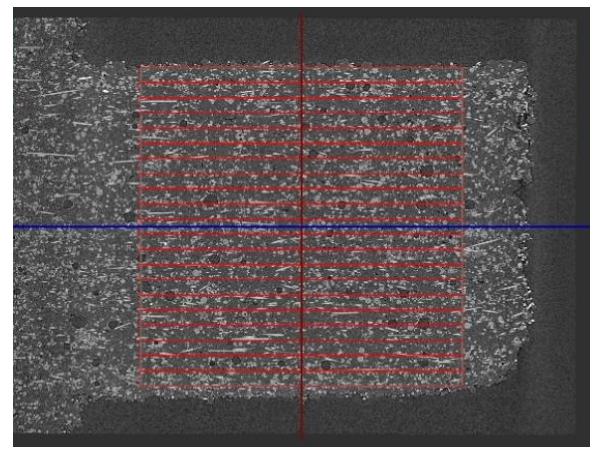

(b)

Figure 3. Illustration of the measuring cube within the sample for tensor calculation (a). The measuring cube is split into a $1 \times 1 \times 21$ mesh (b).

\section{Results}

\subsection{Powder Analysis}

The SEM images in Figure 4 show that the fillers are added to the powder and are not integrated into the polymer particles. The length of fibres varies up to a maximum of $500 \mu \mathrm{m}$, while the fibre diameters have a constant value of about $15 \mu \mathrm{m}$. Furthermore, the diameter of the beads varies up to $80 \mu \mathrm{m}$. The potato-shaped geometry of the powder particles is a result of the fabrication process of the powder. Farsoon reported that the PA6 powder is fabricated by precipitation of a dissolved PA6 [12]. Additionally, small spherical additives are commonly added to the powder, which act as separating agents to decrease the particle-particle attractive forces for a better powder flowability [13]. In these SEM images, dust on the particles is identifiable, which could also support the flowability.

The dynamic DSC analysis of the powder is illustrated in Figure 5. The melting and crystallisation peaks are clearly defined, which allows one to derive the optimum processing parameters. The chosen build temperature of $210^{\circ} \mathrm{C}$ is $6{ }^{\circ} \mathrm{C}$ lower than the onset melt temperature $\left(216^{\circ} \mathrm{C}\right)$. The fillers do not significantly influence the melting curve. The temperature of the cavity walls and piston plate was set to $180^{\circ} \mathrm{C}$ to allow the melted material to cool down as slowly as possible, so as to avoid warpage effects. Furthermore, it can be observed that the crystallisation is initiated at higher temperatures $\left(195^{\circ} \mathrm{C}\right)$ in comparison to non-filled materials (BASF Ultrasint ${ }^{\circledR}$ PA6: $188^{\circ} \mathrm{C}$ ). The glass fibres, glass beads, carbon black and other additives act as crystallisation nuclei. Depending on the size and geometry of the crystallisation nucleus, the crystalline structure is influenced. 


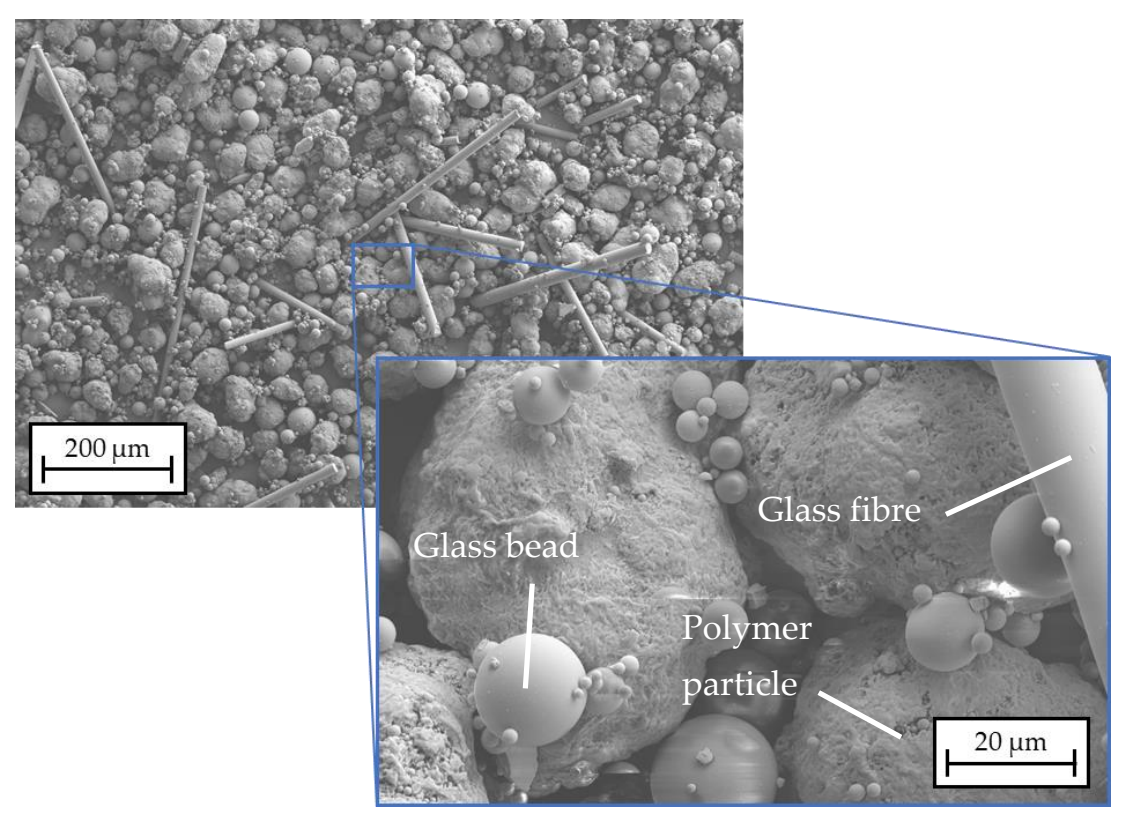

Figure 4. Powder composition of the investigated reinforced PA6 powder.

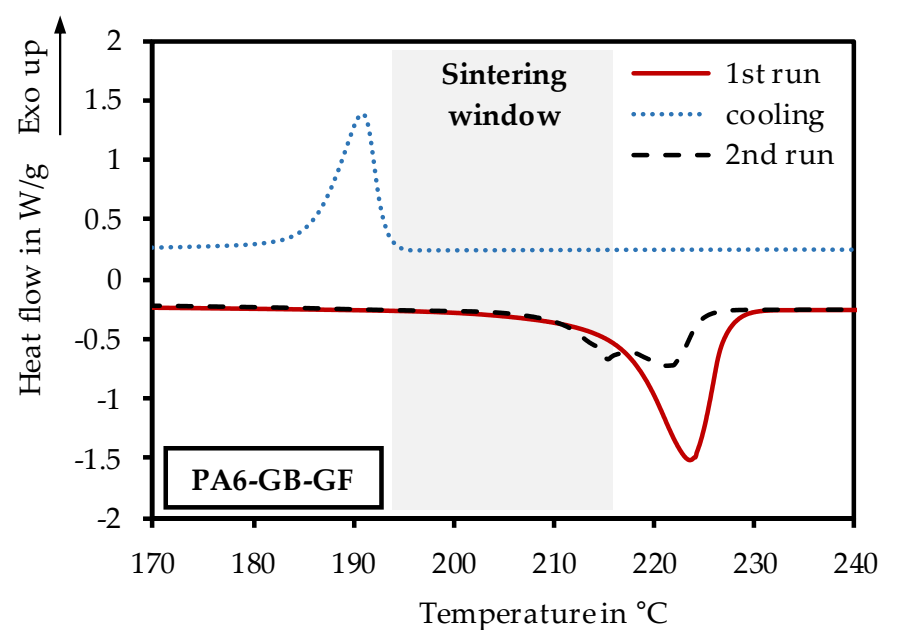

Figure 5. Characterisation of the melting and crystallisation behaviour of PA6-GB-GF powder.

\subsection{Process Influence on the Mechanical Properties}

For the evaluation of influences on the mechanical properties, the modulus of elasticity is chosen as a key factor. The analysis of the DoE shows that the values of the modulus of elasticity follow a normal distribution ( $p$-value $<0.005)$. All factors show significant effects (significance level: 1.98). LT and LP show similar effects, with effect values above 7.2. RS shows low significance with 2.5. Furthermore, the correlation of LT and LP has an effect of 5.5. This high significance is due to the impact of both parameters on to the resulting volumetric energy density. The other correlations show no significance.

Figure 6 illustrates the results of mechanical testing. The result shows averaged values for the modulus of elasticity. As observed at effect significance, RS has only a little influence on the sample's modulus of elasticity. There is a maximum at medium setting, while the low and high setting leads to lower values. In total, the impact of RS is hardly measurable. LT and LP, on the other hand, show higher impacts. The higher LT, the lower is the modulus of elasticity. The opposite trend is observed for LP. A high increase from low to medium setting can be observed. At the medium setting, the maximum is achieved and remains on this level with a small decrease in the high setting. Both effects can be explained by the resulting volumetric energy density. The lower LT and/or the higher LP, the higher 
is the resulting energy. The higher modulus of elasticity can be found with the better layer-to-layer interconnection [9]. For the influence of fibre orientation on the mechanical properties, the orientation tensors in the following chapters are necessary.

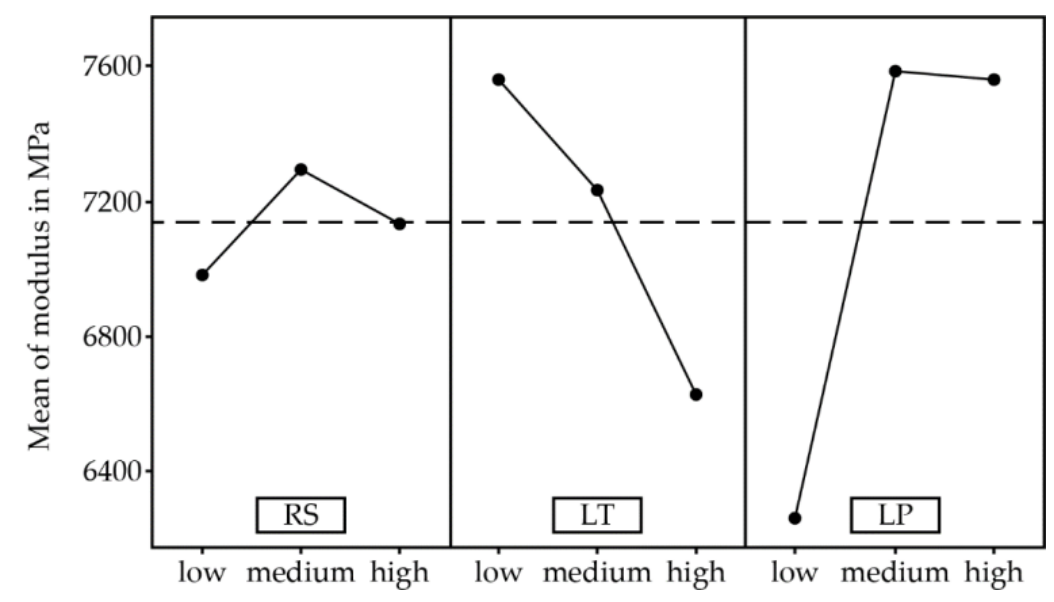

Figure 6. Effect of processing parameters on the modulus of elasticity of the produced samples.

\subsection{Sample Selection for CT Investigation}

The analysis of the modulus of elasticity itself does not directly allow a conclusion as regards the fibre orientation. Therefore, samples for the $\mathrm{CT}$ are selected, which display significant differences in their mechanical values. The selected samples are summarised in Table 3. The CT1 cube reflects the parameter combination with the highest modulus of elasticity of $8154 \mathrm{MPa}$, and is taken as the reference. The selection for the other $\mathrm{CT}$ cubes is reasoned to be below. In addition, the resulting volumetric energy density for each sample is calculated.

The selected samples also vary in their tensile strength and elongation at break. The values are summarised in Figure 7. The results show a correlation with the resulting laser energy density, whereas the three settings with sufficiently high laser energy density (CT1 $\left(197 \mathrm{~J} / \mathrm{cm}^{3}\right), \mathrm{CT} 3\left(236 \mathrm{~J} / \mathrm{cm}^{3}\right)$ and CT5 $\left.\left(197 \mathrm{~J} / \mathrm{cm}^{3}\right)\right)$ lead to high strengths as well as elongations. Lower mechanical properties are observed for CT2 $\left(169 \mathrm{~J} / \mathrm{cm}^{3}\right)$ and CT4 $\left(87 \mathrm{~J} / \mathrm{cm}^{3}\right)$ if the laser energy density is much lower. For high layer-to-layer interconnection, the threshold value can be found between $169 \mathrm{~J} / \mathrm{cm}^{3}$ and $197 \mathrm{~J} / \mathrm{cm}^{3}$. In addition, the recoating speed seems to influence the reproducibility. The setting with the lowest recoating speed (CT5) shows the lowest standard deviation of $1.72 \mathrm{MPA}(2.1 \%)$ for the tensile strength.

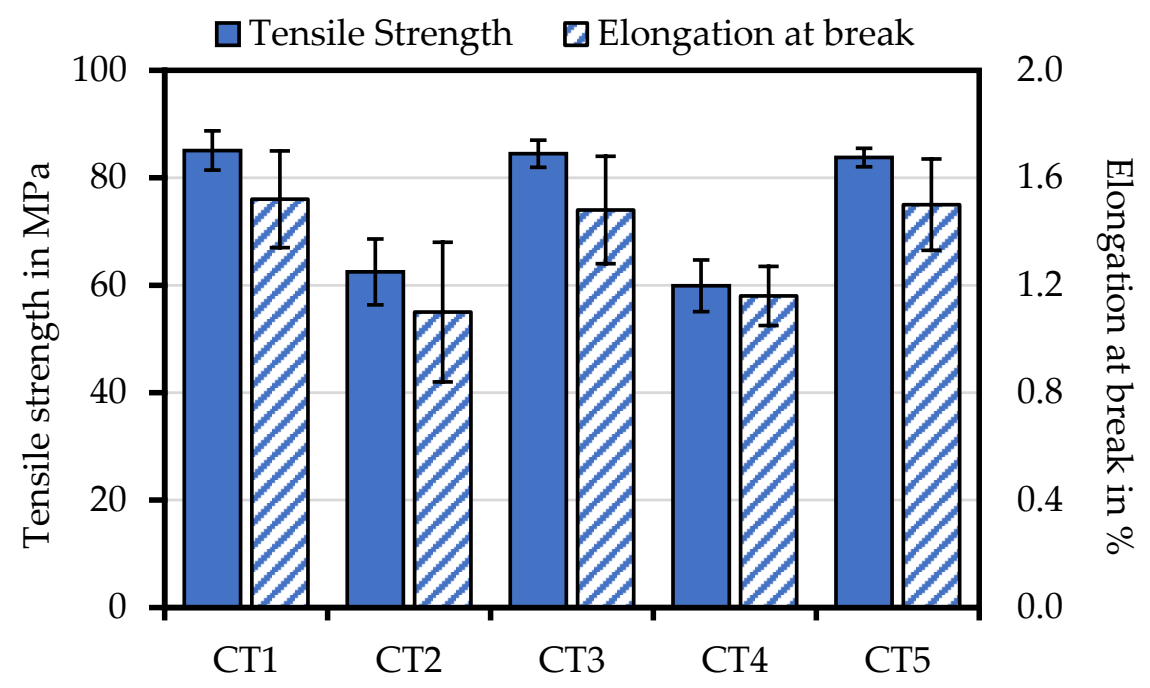

Figure 7. Effects of the varied parameters on tensile strength and elongation at break. 
Table 3. Selection of samples for computed tomography scans.

\begin{tabular}{|c|c|c|c|c|c|c|}
\hline Label & RS & LT & $\mathbf{L P}$ & $\begin{array}{l}\text { Resulting Energy } \\
\text { Density in } \mathrm{J} / \mathrm{cm}^{3}\end{array}$ & $\begin{array}{c}\text { Modulus of } \\
\text { Elasticity in MPa }\end{array}$ & Selection Criterion \\
\hline $\begin{array}{c}\mathrm{CT} 1 \\
\text { (reference) }\end{array}$ & high & medium & high & 197 & 8154 & Maximum modulus of elasticity \\
\hline $\begin{array}{c}\text { CT2 } \\
\text { (Appendix A: Figure A1) }\end{array}$ & high & high & high & 169 & 6881 & Impact layer thickness high \\
\hline $\begin{array}{c}\text { CT3 } \\
\text { (Appendix A: Figure A2) }\end{array}$ & high & low & high & 236 & 7695 & Impact layer thickness low \\
\hline $\begin{array}{c}\text { CT4 } \\
\text { (Appendix A: Figure A3) }\end{array}$ & high & medium & low & 87 & 6417 & Impact laser energy \\
\hline $\begin{array}{c}\text { CT5 } \\
\text { (Appendix A: Figure A4) }\end{array}$ & low & medium & high & 197 & 7548 & Impact recoating speed \\
\hline
\end{tabular}




\subsection{Fibre Orientation Dependent on Processing Parameters}

The evaluation of CT1 shows that fibres tend to align in the powder recoating direction (Figure 8a). The SLS's typical rough surface can be detected all around the edge areas. At the right bottom edge, curling effects can be observed, which on a macroscopic view lead to a smooth transition between right side and bottom side. Next to the aligned glass fibres, glass beads of different sizes, as well as shrinkage-related voids, occur inside the part. Furthermore, the top layers show less filler particles than the bottom layers. Another difference in the comparison of the top/bottom layer is seen in the melting behaviour of the particles. The first layer of the sample displays partially melted powder particles, while particles in the top layer melted almost completely. This effect can be explained via the layer's temperature distribution before a new layer of powder is added. While in the first layer, the temperature of the applied powder corresponds to the build temperature, the existing temperature is much lower in comparison to the top layer, where a higher temperature results from the heat of previously melted layers. Beyond that, Figure $8 \mathrm{~b}$ shows the fibre orientation in the $\mathrm{X}$ and $\mathrm{Y}$ direction. Fibres are mainly orientated in the $X$ direction. In addition, the orientation in the $Y$ direction is more highly detectable than orientation in the $\mathrm{Z}$ direction.

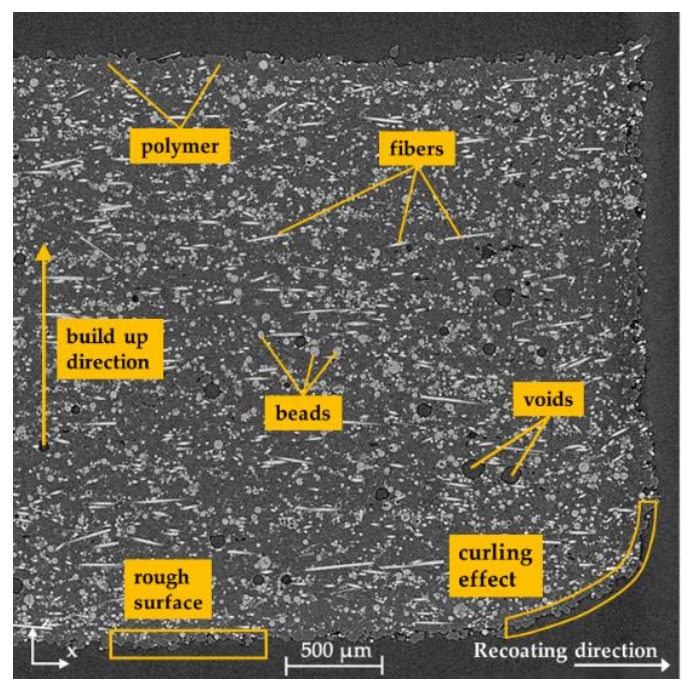

(a)

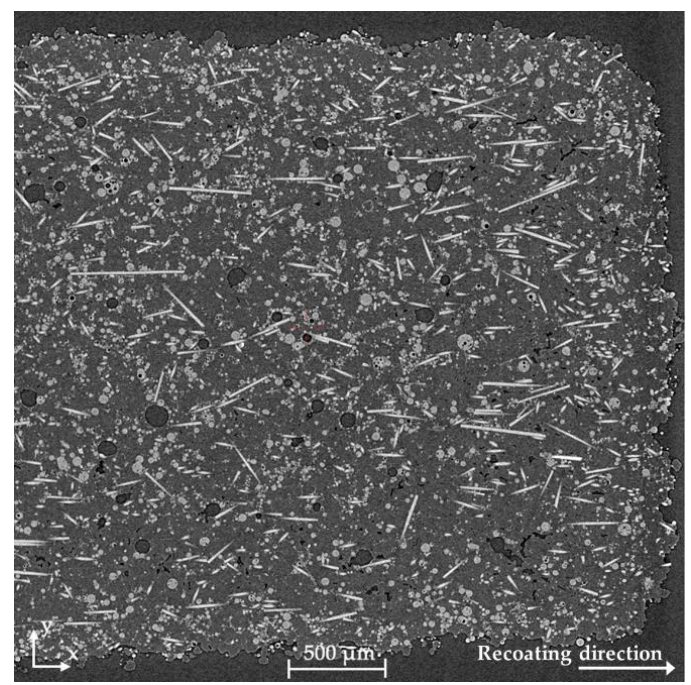

(b)

Figure 8. 2D cross-sectional slice from the CT scan of CT1. Fibre orientation in (a) XZ plane and (b) XY plane. Parameters: RS $=288 \mathrm{~mm} / \mathrm{s}, \mathrm{LT}=120 \mu \mathrm{m}$ and $\mathrm{LP}=36 \mathrm{~W}(\mathrm{CT} 1)$.

The calculation of the tensors for CT1 is illustrated in Figure 9. As already seen in the qualitative results above, the $X$ alignment is the dominating tensor, followed by $Y$ and $Z$. The value of the $X$ tensor varies from 0.49 to 0.58 along the grid elements. The $Y$ tensors shows a value of 0.28 , and $Z$ of 0.18 .

Mean values of the 21 tensor measuring ranges are calculated for each orientation tensor and CT scan sample. The mean values of all investigated tensors are summarised in Figure 10. Except CT3, the tensors show similar characteristics for the $\mathrm{X}, \mathrm{Y}$ and $\mathrm{Z}$ directions. With a higher value of approximately 0.04 , the $\mathrm{X}$ tensor of $\mathrm{CT} 3$ shows a greater orientation of fibre in the recoating direction.

The CT3 image in Figure 11 shows similar results as that of CT1. Despite the $39 \mathrm{~J} / \mathrm{cm}^{3}$ higher energy input, voids are still visible. The slightly increased fibre orientation is not recognisable from this single image, but could be measured by tensor evaluation across all layers. Due to the lower layer thickness, more fibres interact with the roller and an increased orientation is the result. Despite the increased laser energy and higher orientation, the modulus of elasticity decreases compared to CT1. At this point two effects overlap: the modulus of elasticity increases due to the fibre orientation in the recoating and tensile test direction, and the high energy density leads to a degradation in the polymer material. 


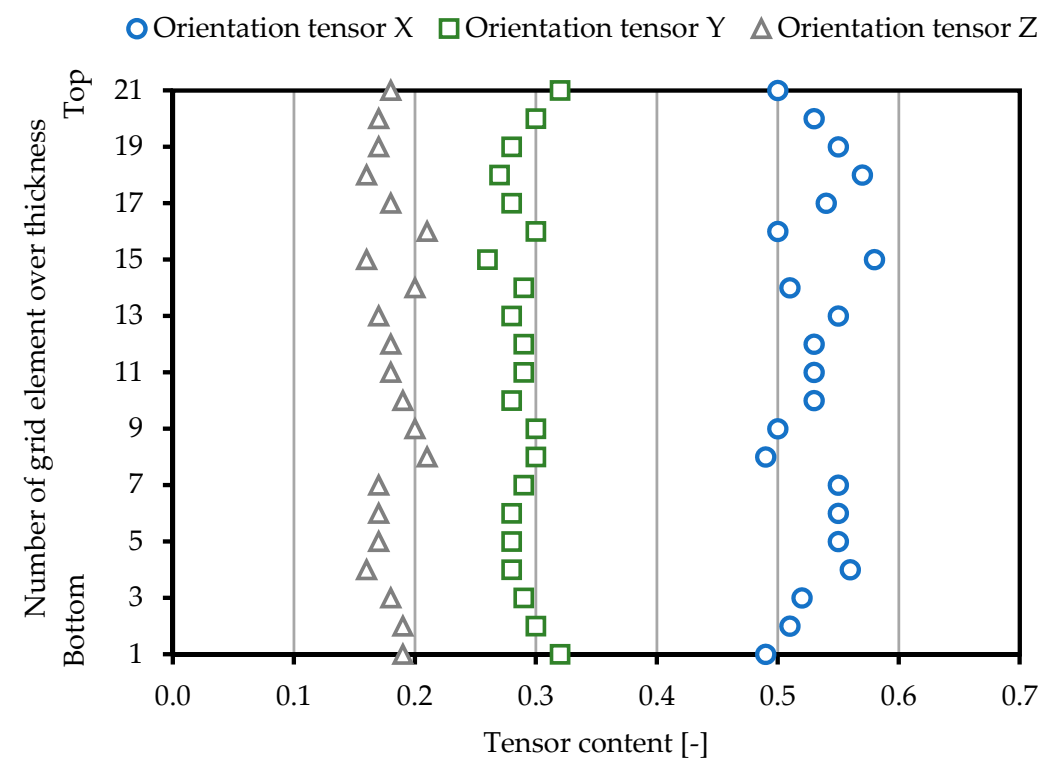

Figure 9. Fibre orientation in CT1 with the parameters RS $=288 \mathrm{~mm} / \mathrm{s}, \mathrm{LT}=120 \mu \mathrm{m}$ and LP $=36 \mathrm{~W}$.

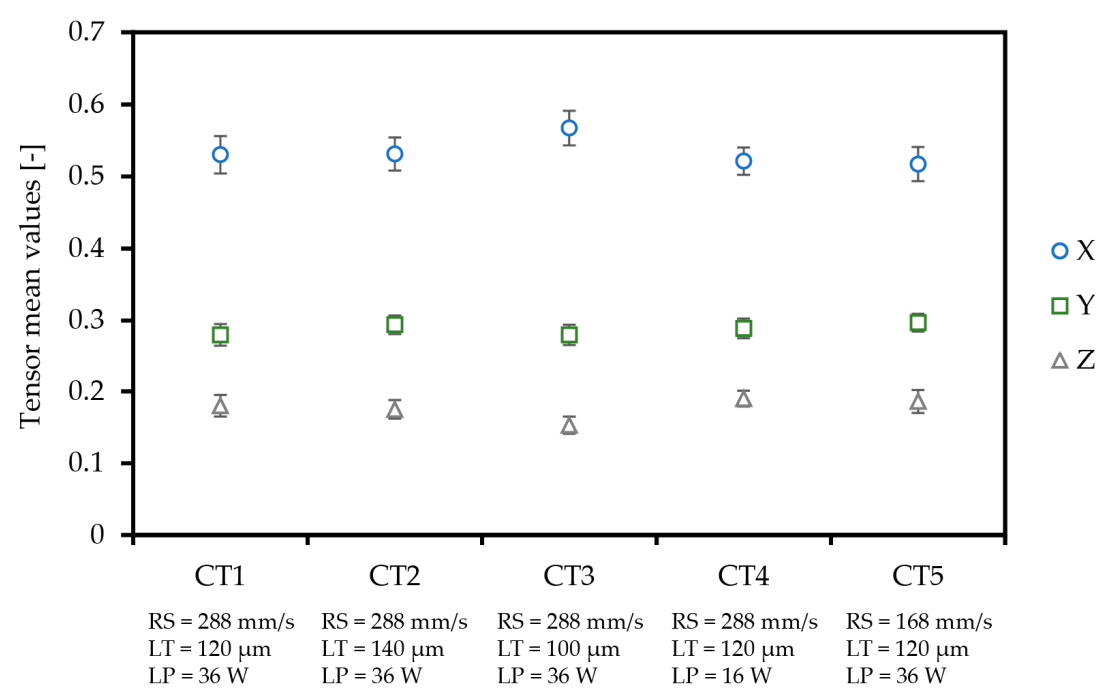

Figure 10. Comparison of all tensor values for all investigated CT scans.

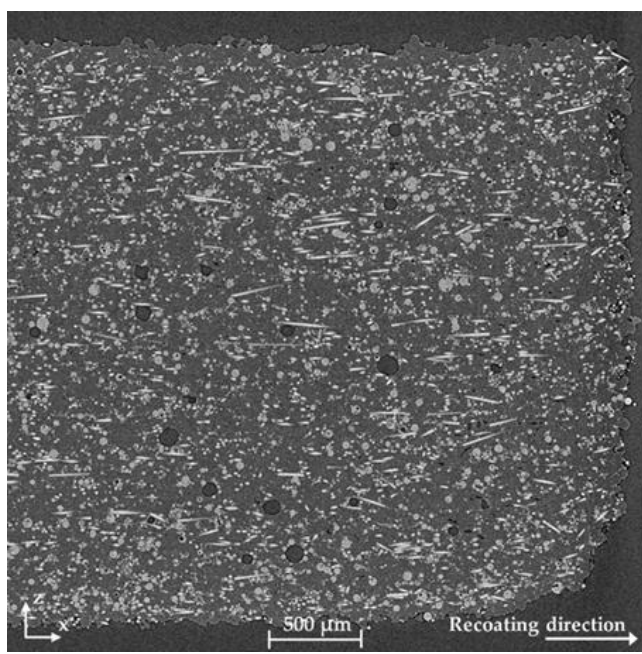

Figure 11. 2D cross-sectional slice from the CT scan of CT1. Parameters: RS $=288 \mathrm{~mm} / \mathrm{s}, \mathrm{LT}=100 \mu \mathrm{m}$ and $\mathrm{LP}=36 \mathrm{~W}$ (CT3). 
Figure 12 illustrates the result of insufficient laser energy in sample CT4. Many voids within the part occur, which reduces the layer-to-layer interconnection. This leads to a low tensile strength of $60 \mathrm{MPa}$ and low elongation at break of $1.2 \%$. In addition, the reduced laser energy leads to a reduction in the curling. Because of the lower melt temperature, the temperature difference between the melt and the recoated powder is comparably low. Therefore, internal stresses are reduced and less curling occurs.

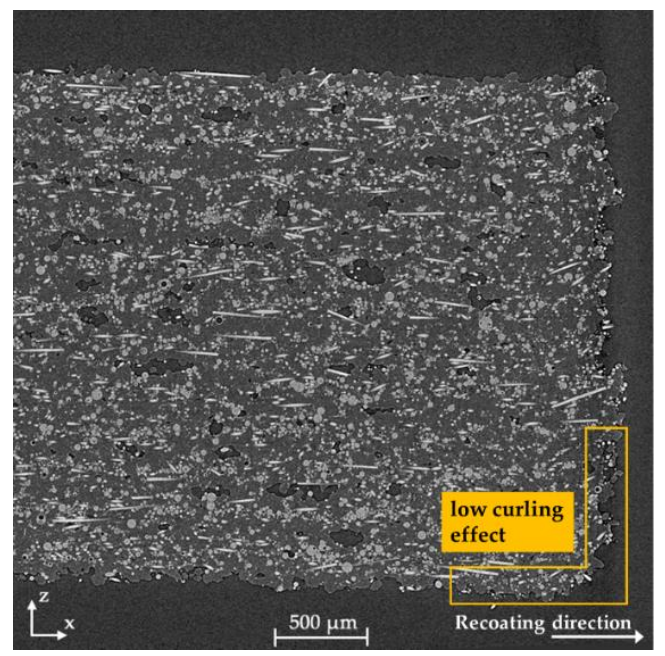

Figure 12. Insufficient laser power leads to defects in the part's material and consequently to a low layer-to-layer interconnection. Parameters: RS $=288 \mathrm{~mm} / \mathrm{s}, \mathrm{LT}=120 \mu \mathrm{m}$ and $\mathrm{LP}=16 \mathrm{~W}(\mathrm{CT} 4)$.

\section{Discussion}

The results of the design of experiment show the high significance of the factors of layer thickness and laser power on the modulus of elasticity. The recoating speed has A low significance. Interactions between the three factors can only be identified for LT and LP. This interaction effect is the result of the dependence of these factors on the resulting energy density. The higher the energy density, the higher the mechanical properties.

The tensor analysis, for instance, shows that the varied laser power does not influence the fibre orientation, as comparing CT1 $\left(197 \mathrm{~J} / \mathrm{cm}^{3}\right)$ and CT4 $\left(87 \mathrm{~J} / \mathrm{cm}^{3}\right)$ shows similar X-tensors $(0.53$ and 0.52). Although the melt viscosity changes with melt temperature, which is directly related to the applied laser power, there is no external force, except gravity, acting on the fibres, which could cause changes in fibre orientation. Lower viscosity can also improve the wetting of the fibres, leading to an improved introduction of load into the fibres and higher part strength, respectively. As such, the effect of higher laser power on part strength results from an improved layer-to-layer interconnection and fibre incorporation, and not necessarily from a change of fibre orientation. This can also be seen in the CT scans, which confirm that the laser energy improves the quality of the layer-to-layer interconnection. Theoretically, perpendicular fibres are able to penetrate several layers ( $Z$ direction) more easily if the viscosity is lower. Nevertheless, as mentioned above there is no additional driving force, related to the laser power, which could cause fibre reorientation. Consequently, the CT analysis shows no influence of the energy density on fibre orientation. Thus, fibres mainly follow a 2D orientation inside a layer.

Variation of layer thickness has two effects: First, the laser energy density is proportionately dependent on the layer thickness. As a consequence, the quality of the layer-to-layer interconnection and fibre wetting is affected in the same manner as described above for the laser power. The second effect is directly related to fibre orientation, whereby lower layer thicknesses result in greater orientation in the recoating direction. By reducing the layer thickness, the recoating roller interacts with more fibres, since even smaller fibres can interact and change orientation. In addition, the shear stress between the top and bottom of each layer, which results from the roller's counter-rotation to the recoating direction, is inversely proportional to the layer thickness. Hence, the driving force causing fibre alignment in the 
recoating direction increases as the layer thickness decreases. For CT3 with a low layer thickness, the $\mathrm{X}$ tensor shows an increase of $7 \%$ in comparison to the reference with a medium setting for the layer thickness. Since spherical beads do not have an influence on the tensors, the alignment of the fibres will be higher than the calculated 7\% suggests. The shown impact of the layer thickness parameter confirms Jansson's [4] findings, with the effect illustrated in Figure 13. Although the layer thickness has a significant effect on fibre orientation, the layer thickness cannot be set too low, otherwise the powder spreading process will be hindered considering the poorer flowability of the composite powders.

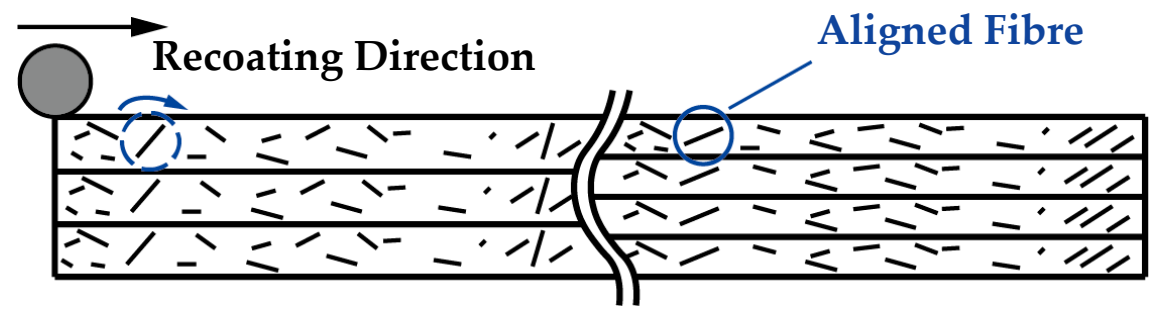

Figure 13. Orientation of fibres due to variation of the layer thickness from a high (left) to a low level (right).

As opposed to the layer thickness, the recoating speed determines the duration of the roller-fibre interaction. Results show that the factor time (interaction between roller and fibre) has little impact on the resulting modulus of elasticity within the chosen settings. The fibre orientation tensors show a slightly higher orientation for the high setting (CT1) in comparison to the low setting (CT5).

\section{Conclusions and Outlook}

The current work indicates that for PA6 powder, filled with $30 \mathrm{wt} . \%$ glass beads and $10 \mathrm{wt} . \%$ glass fibres, the modulus of elasticity can vary strongly (6417-8154 MPa) in dependence on the chosen process parameters. All these variations are related to the applied laser energy density, which was either varied directly by the setting of the applied laser power, or indirectly by layer thickness. The dominant effect can be found in an improved layer-to-layer interconnection and fibre wetting, which is related to the melt viscosity as a result of the varied laser energy density. In addition, reduced layer thickness leads to a slightly higher $(7 \%)$ fibre alignment in the recoating direction, with smaller fibres now in interaction with the roller and thus aligning in the roller direction. In addition, a change in the melt viscosity due to variation of the energy density does not seem to affect fibre orientation.

No significant impact in terms of fibre orientation related to the parameters of recoating speed and laser power was found. With their apparently low influence on fibre orientation during the processing of fibre-filled PA6 powder, these parameters can be used to focus on process robustness. Conclusively, since the degree of anisotropy in fibre orientation can be influenced by the layer thickness, it would be possible to induce locally adjusted stiffness and anisotropic mechanical behaviour with changes in layer thickness, in order to improve part performance. This study illustrates the need to understand and optimise processing parameters so as to achieve the best mechanical properties of the laser sintered parts, especially with fibre- and bead-reinforced powders.

This study dealt with the effect of selective laser sintering on the fibre orientation in manufactured components. Based on the results shown, it can be assumed that the layer height as well as the laser power have influence on the material structure in the manufactured test specimens. Therefore, the melting process and the herein-induced properties (e.g., porosity, degree of crystallinity, temperature profile) can be investigated in detail in future work.

Author Contributions: Conceptualisation, T.H.; methodology, T.H.; validation, T.H. and M.S.; formal analysis, T.H.; investigation, T.H. and M.S.; data curation, T.H. and M.S.; writing-original draft preparation, T.H.; writing-review and editing, M.S.; S.R.R., G.H. and P.M.; visualization, T.H.; supervision, S.R.R., G.H. and P.M. All authors have read and agreed to the published version of the manuscript.

Funding: This research received no external funding. 
Acknowledgments: The authors gratefully acknowledge Farsoon Europe for the technical support and provision of the investigated reinforced PA6 material (FS6140GF). Any opinions, findings, conclusions or recommendations expressed in this study are those of the authors and do not necessarily reflect the views of Farsoon Europe.

Conflicts of Interest: The authors declare no conflict of interest.

\section{Appendix A}

\section{CT Scans and Fibre Orientations Tensors}
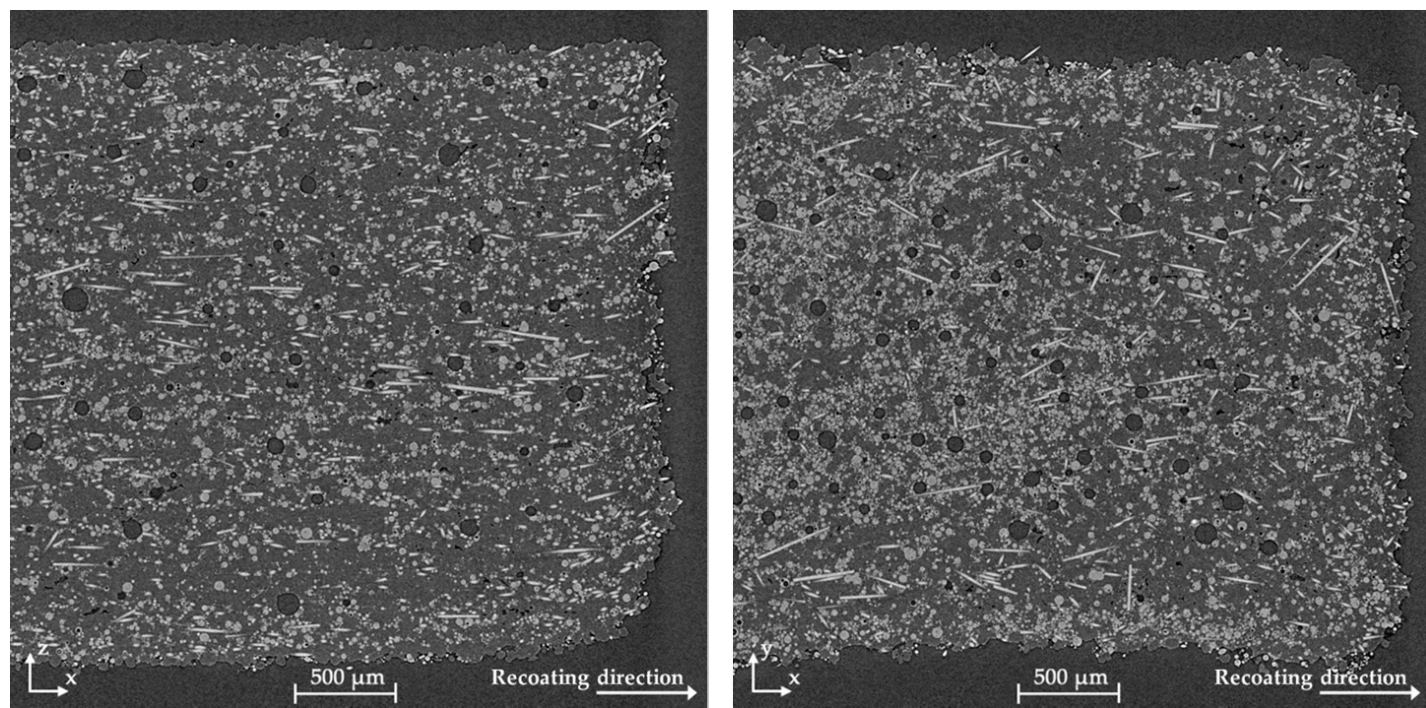

O Orientation tensor $\mathrm{X} \square$ Orientation tensor $\mathrm{Y} \Delta$ Orientation tensor $\mathrm{Z}$

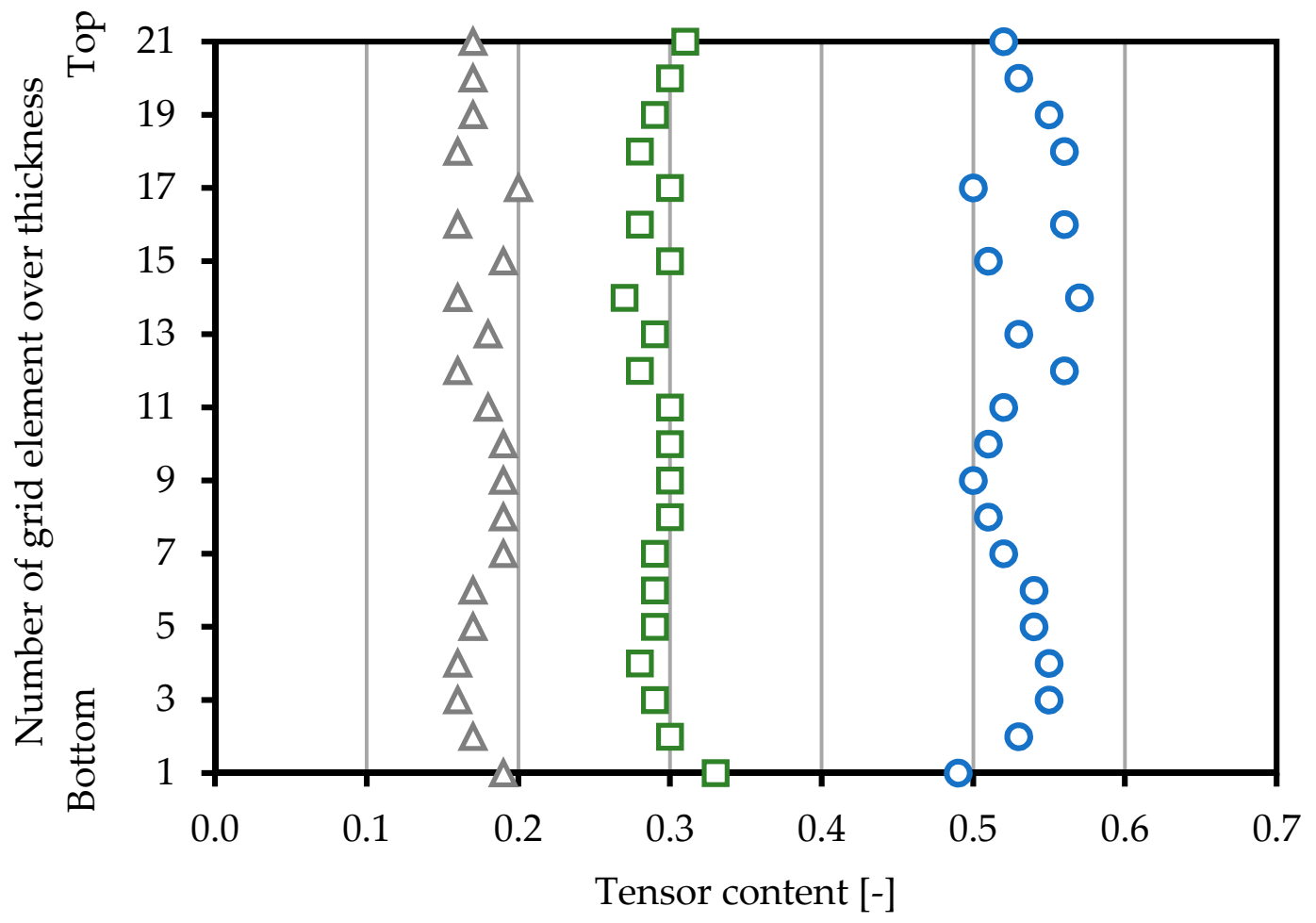

Figure A1. CT2-RS $=288 \mathrm{~mm} / \mathrm{s}, \mathrm{LT}=140 \mu \mathrm{m}, \mathrm{LP}=36 \mathrm{~W}$. 

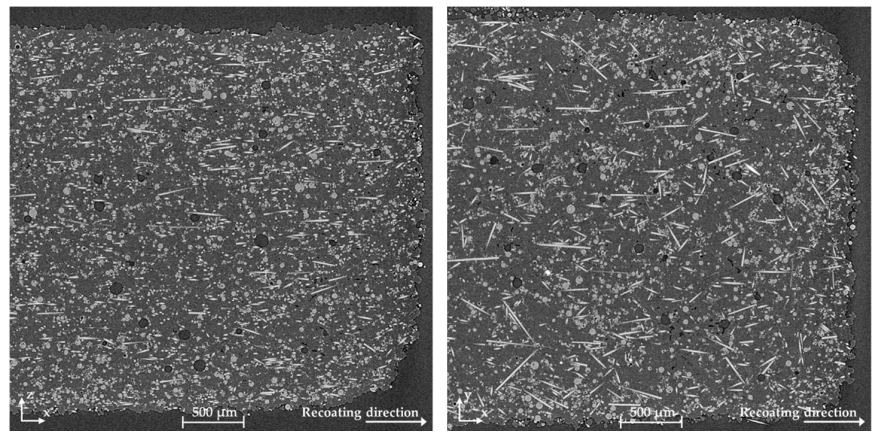

O Orientation tensor $\mathrm{X} \square$ Orientation tensor $\mathrm{Y} \Delta$ Orientation tensor $\mathrm{Z}$

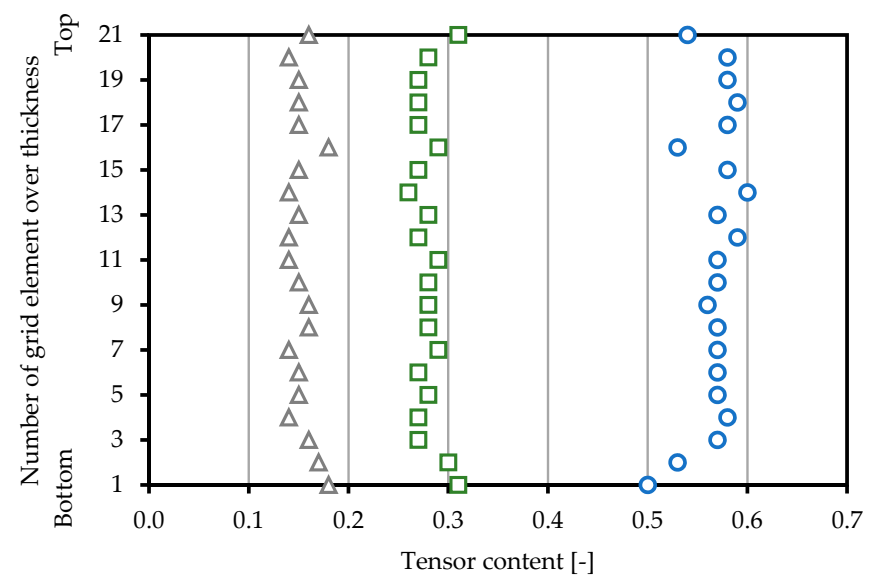

Figure A2. CT3-RS $=288 \mathrm{~mm} / \mathrm{s}, \mathrm{LT}=100 \mu \mathrm{m}, \mathrm{LP}=36 \mathrm{~W}$.
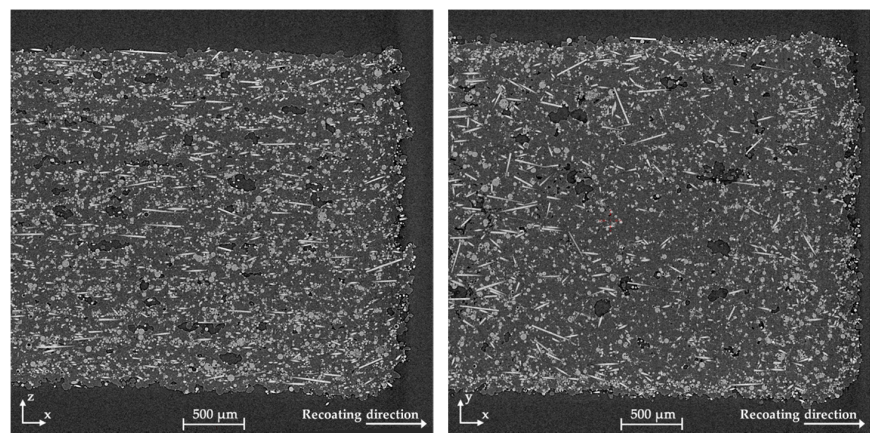

O Orientation tensor $\mathrm{X} \square$ Orientation tensor $\mathrm{Y} \Delta$ Orientation tensor $\mathrm{Z}$

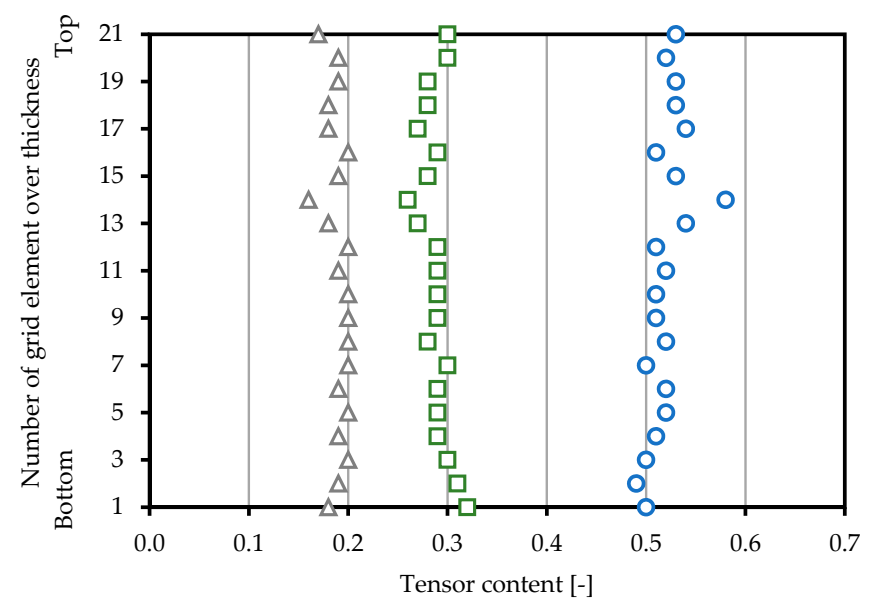

Figure A3. CT4-RS $=288 \mathrm{~mm} / \mathrm{s}, \mathrm{LT}=120 \mu \mathrm{m}, \mathrm{LP}=16 \mathrm{~W}$. 

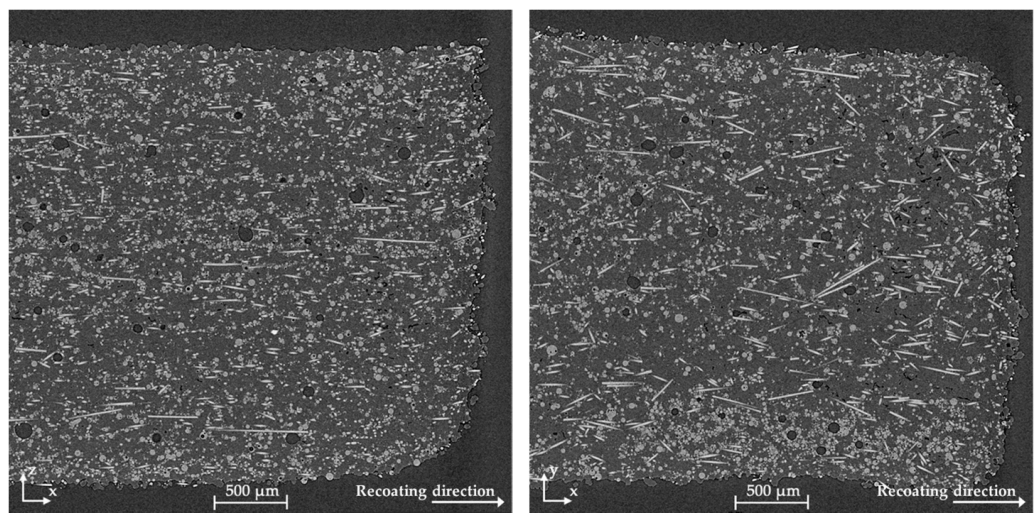

O Orientation tensor $\mathrm{X} \square$ Orientation tensor $\mathrm{Y} \Delta$ Orientation tensor $\mathrm{Z}$

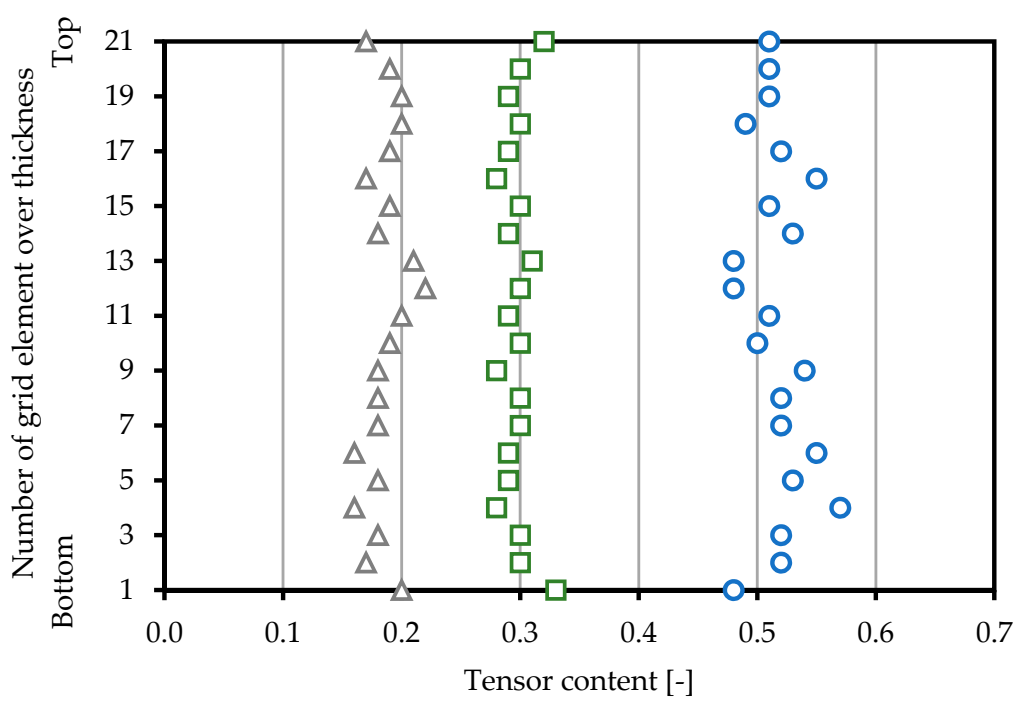

Figure A4. CT5-RS $=168 \mathrm{~mm} / \mathrm{s}, \mathrm{LT}=120 \mu \mathrm{m}, \mathrm{LP}=36 \mathrm{~W}$.

\section{References}

1. Bay, R.S.; Tucker, C.L. Fiber orientation in simple injection molding. Part II: Experimental results. Polym. Compos. 1992, 13, 332-341. [CrossRef]

2. Gandhi, U.N.; Goris, S.; Osswald, T.A.; Song, Y.-Y. Discontinuous Fiber-Reinforced Composites: Fundamentals and Applications; Hanser: Munich, Germany, 2020.

3. Colon, J.L.; Heckner, T.; Chrupala, A.; Pollock, J. Experimental study of particle migration in polymer processing. Polym. Compos. 2019, 40, 2165-2177. [CrossRef]

4. Jansson, A.; Pejryd, L. Characterisation of carbon fibre-reinforced polyamide manufactured by selective laser sintering. Compos. Struct. 2016, 9, 7-13. [CrossRef]

5. Arai, S.; Tsunoda, S.; Yamaguchi, A.; Ougizawa, T. Effect of anisotropy in the build direction and laser-scanning conditions on characterization of short-glass-fiber-reinforced PBT for laser sintering. Opt. Laser Technol. 2019, 113, 345-356. [CrossRef]

6. Schmid, M. Selektives Lasersintern (SLS) Mit Kunststoffen: Technologie, Prozesse und Werkstoffe; Hanser: Munich, Germany, 2015; p. 178.

7. Beitz, S.; Uerlich, R.; Bokelmann, T.; Diener, A.; Vietor, T.; Kwade, A. Influence of powder deposition on powder bed and specimen properties. Materials 2019, 12, 297. [CrossRef]

8. Drexler, M.; Greiner, S.; Lexow, M.; Lanzl, L.; Wudy, K.; Drummer, D. Selective laser melting of polymers: Influence of powder coating on mechanical part properties. J. Polym. Eng. 2018, 38, 667-674. [CrossRef]

9. Heckner, T.; Raisch, S.R.; Huelder, G.; Middendorf, P. The Influence of Laser Power Variation on SLS-Printed PA6 Parts and the Resulting Effect on The Parts' Long-Term Properties. In Proceedings of the ANTEC 2020: The Virtual Edition, Brookfield, CT, USA, 29 March-2 April 2020. 
10. Lanzl, L.; Wudy, K.; Drummer, D. The effect of short glass fibers on the process behavior of polyamide 12 during selective laser beam melting. Polym. Test. 2020, 83, 106313. [CrossRef]

11. Zhu, W.; Yan, C.; Shi, Y.; Wen, S.; Liu, J.; Wei, Q.; Shi, Y. A novel method based on selective laser sintering for preparing high-performance carbon fibres/polyamide12/epoxy ternary composites. Sci. Rep. 2016, 6, 33780. [CrossRef]

12. Simon, D.; FARSOON Europe GmbH, Stuttgart, Germany. Personal communication, 2020.

13. Wudy, K. Alterungsverhalten Von Polyamid 12 Beim Selektiven Lasersintern. Ph.D. Thesis, University of Erlangen-Nuremberg, Erlangen, Germany, 2017.

(C) 2020 by the authors. Licensee MDPI, Basel, Switzerland. This article is an open access article distributed under the terms and conditions of the Creative Commons Attribution (CC BY) license (http://creativecommons.org/licenses/by/4.0/). 